\title{
ON CERTAIN PARTIAL DIFFERENTIAL OPERATORS OF FINITE ODD TYPE
}

\author{
A. ALEXANDROU HIMONAS
}

\begin{abstract}
Let $P$ be a linear partial differential operator of order $m \geq 1$ with real-analytic coefficients defined in $\Omega$, an open set of $\mathbb{R}^{n}$, and let $\gamma$ be in the cotangent space of $\Omega$ minus the zero section. If $P$ is of odd finite type $k$ and if the Hörmander numbers are $1=k_{1}<k_{2}, k_{2}$ odd, then $P$ is analytic hypoelliptic at $\gamma$. These operators are not semirigid.
\end{abstract}

\section{INTRODUCTION}

In this paper we present a microlocal analytic hypoellipticity result for a class of linear partial differential operators of finite type with real-analytic coefficients. The assumption is that the type of the operator is an odd positive integer $k$ and that there are two Hörmander numbers $k_{1}, k_{2}$ with $1=k_{1}<k_{2}$ and $k_{2}$ is odd. The main difference between this result and previous results obtained by the author is that the operators here may not be semirigid. Therefore we view it as a partial result of the more general problem of giving necessary and sufficient conditions for microlocal analytic hypoellipticity of operators of finite type. For $C R$ structures a version of this problem has been recently solved by Tumanov [10] (sufficient conditions) and Baouendi-Rothschild [1] (necessary conditions). In the analytic case the necessity was proved in [2].

The condition used in [10] is a condition at a point in the base space called the minimality condition. An analytic $C R$ structure is minimal at a point if it is of finite type at this point . For $C^{\infty} C R$ structures finite type implies minimality but not vice versa. The finite type condition used in this paper is microlocal.

The new difficulty that arises in the proof of this result is that the phase function may contain terms of degree less than $k$ which is the degree of the terms that result from the type of the operator. To make these lower degree terms negligible, we choose a special good contour and we use a more general sufficient condition than the one used in [6].

Received by the editors December 18, 1989.

1980 Mathematics Subject Classification (1985 Revision). Primary 35H05; Secondary 58G15.

The author was partially supported by NSF. 
The author would like to thank J. E. Fornaess for suggesting the proof of Lemma 2.3.

\section{Definitions and Statement of Result}

Let $P=P(y, D)$ be a linear partial differential operator (p.d.o.) of order $m \geqslant 1$ with complex-valued real-analytic coefficients defined in an open neighborhood $\Omega$ of $y_{0} \in \mathbb{R}^{n}$. We denote by $P_{m}(y, \eta)$ its principal symbol with $p_{1}(y, \eta)=\Re P_{m}(y, \eta)$ the real part and $p_{2}(y, \eta)=\Im P_{m}(y, \eta)$ the imaginary part. Also, let $\gamma=\left(y_{0}, \eta_{0}\right) \in T^{*} \Omega-0$ be a characteristic point for $P$ and assume that $P$ is of principal type at $\gamma$, i.e., $P_{m}(\gamma)=0$ and $\operatorname{grad}_{\eta} P_{m}(\gamma) \neq 0$.

If $I=\left(i_{1}, \ldots, i_{j}\right)$ is a sequence of $|I|=j$ elements which are either 1 or 2 then we denote by

$$
p_{I}=\left\{p_{i_{j}},\left\{p_{i_{j-1}}, \ldots,\left\{p_{i_{2}}, p_{i_{1}}\right\} \ldots\right\}\right\}
$$

the repeated Poisson bracket of $p_{1}$ and $p_{2}$ associated to $I$.

The p.d.o. is said to be of finite type $k=k(\gamma)$ at $\gamma$ (Egorov [4], Hörmander [7], Kohn [8]) if $p_{I}(\gamma)=0$ for all $I$ with $|I|<k$, and if $p_{I}(\gamma) \neq 0$ for all $I$ with $|I|=k$.

Now we shall recall the definition of Hörmander numbers (see also [6]) for a p.d.o. $P$ of finite type $k, k \geqslant 2$. Let $\lambda=\eta_{1} \frac{\partial}{\partial \eta_{1}}+\cdots+\eta_{n} \frac{\partial}{\partial \eta_{n}}$ and $V_{0}=$ $\operatorname{span}\{\lambda(\gamma)\}$ in $T_{\gamma}\left(T^{*} \mathbb{R}^{n}\right)$. We define

$$
k_{1}=k_{1}(\gamma)=\min \left\{|I|:|I| \leq k / 2 \text { and } H_{p_{I}}(\gamma) \notin V_{0}\right\} \text {. }
$$

Here $H_{p_{I}}$ is the Hamilton vector field of $p_{I}$. We let $V_{1}=\operatorname{span}\left\{H_{p_{I}}(\gamma)\right.$ : $\left.|I| \leqslant k_{1}, \lambda(\gamma)\right\}$, and we define the multiplicity $d_{1}$ of $k_{1}$ by $d_{1}=\operatorname{dim} V_{1}-$ $\operatorname{dim} V_{0}$. We proceed inductively. We assume that the numbers $k_{1}, \ldots, k_{\nu}$, their multiplicities $d_{1}, \ldots, d_{\nu}$, and the spaces $V_{1}, \ldots, V_{\nu}$ have been defined and we define

$$
\begin{gathered}
k_{\nu+1}=\min \left\{|I|:|I| \leq k / 2 \text { and } H_{p_{I}}(\gamma) \notin V_{\nu}\right\} \\
V_{\nu+1}=\operatorname{span}\left\{H_{p_{I}}(\gamma):|I| \leq k_{\nu+1}, \lambda(\gamma)\right\} \quad \text { and } d_{\nu+1}=\operatorname{dim} V_{\nu+1}-\operatorname{dim} V_{\nu} .
\end{gathered}
$$

The Hörmander numbers $k_{1}, \ldots, k_{r}$ are the numbers defined above, counted with their multiplicities. It is easy to see that the assumption that $P$ is of principal type at $\gamma$ implies that $k_{1}=1$.

Now we shall state our result.

Theorem 1.1. Let $P$ be a linear p.d.o. with real-analytic coefficients defined in $\Omega$, an open set of $\mathbb{R}^{n}$, and $\gamma \in T^{*} \Omega-0$. If $P$ is of odd finite type $k$ and if the Hörmander numbers are $1=k_{1}<k_{2}, k_{2}$ odd, then $P$ is analytic hypoelliptic at $\gamma$.

We recall that $P$ is analytic hypoelliptic at $\gamma$ iff $u$ is analytic at $\gamma$ whenever $P u$ is analytic at $\gamma$. A distribution $u$ defined near $y_{0} \in \mathbb{R}^{n}$ is said to be analytic at $\gamma$ (see Sjöstrand [9]) iff

$$
\left|\int_{\mathbb{R}^{n}} e^{-i x \eta-|\eta|(x-y)^{2}} \chi(x) u(x) d x\right| \leq c e^{-|\eta| / c}
$$


for all $y$ near $y_{0}$ and all $\eta$ in a conic neighborhood of $\eta_{0}$. Here $\chi$ is in $C_{0}^{\infty}\left(\mathbb{R}^{n}\right)$ and equal to 1 near $y_{0}$.

The Hörmander numbers $k_{1}, k_{2}$, and $k$ are invariant under a symplectic change of coordinates, since they are defined by the repeated Poisson brackets of $p_{1}$ and $p_{2}$ and since symplectic changes of coordinates preserve Poisson brackets. By straightforward computations one can show that these numbers are also invariant under a multiplication by an elliptic symbol.

If $P$ is a vector field in $\mathbb{R}^{3}$ then a typical example that Theorem 1.1 applies is

$$
P=\frac{\partial}{\partial y_{1}}+i\left[y_{1}^{k_{2}-1} \frac{\partial}{\partial y_{2}}+p_{k-1}\left(y_{1}, y_{2}\right) \frac{\partial}{\partial y_{3}}\right],
$$

where $k$ and $k_{2}$ are positive odd integer with $1<k_{2}<k / 2$, and $p_{k-1} \neq 0$ is a homogeneous polynomial of weight $k-1$ for the weights $\left(k_{1}, k_{2}\right)$. Here $k_{1}=1$ and it corresponds to the variable $y_{1}, k_{2}$ corresponds to the variable $y_{2}$, and $k$ corresponds to the variable $y_{3}$. The point $\gamma=\left(y_{0}, \eta_{0}\right)$, where $y_{0}=(0,0,0)$ and $\eta_{0}=(0,0, \pm 1)$. By Theorem 1.1 it follows that $P$ is analytic hypoelliptic at $\gamma$. In fact, the vector field $P$ above is analytic hypoelliptic at 0 since at the characteristic points of the form $\gamma=\left(0, \eta_{0}\right), \eta_{0}=\left(0, \eta_{2}, \eta_{3}\right)$ with $\eta_{2} \neq 0, P$ is analytic hypoelliptic by Theorem 1.4 in [6]. Note when $\eta_{0}=(0,0, \pm 1)$ then $P$ is not semirigid at $\gamma$.

\section{Proof of Theorem 1.1}

We start by reducing the p.d.o. $P$ microlocally near $\gamma$ to a classical analytic pseudodifferential operator of order one, which we will denote again with $P$, with principal symbol in the form

$$
p(y, \eta)=\eta_{1}+i p_{2}\left(y, \eta^{\prime}\right), \quad \eta=\left(\eta_{1}, \eta^{\prime}\right),
$$

where $p_{2}$ is a holomorphic function, positively homogeneous of degree one in $\eta^{\prime}$ and

$$
\gamma=\left(y_{0}, \eta_{0}\right), \quad y_{0}=(0, \ldots, 0) \in \mathbb{R}^{n}, \eta_{0}=(0, \ldots, 1) \in \mathbb{R}^{n} .
$$

This reduction is standard for operators of principal type (see [5]) and at the level of principal symbols it is done by using the implicit function theorem and a multiplication by an elliptic symbol.

If, in addition, the Hörmander numbers of the operator $P$ are as in the assumptions of Theorem 1.1 then by Proposition 2.2 in [6] we obtain the following normal form for the principal symbol of our p.d.o. $P$.

Lemma 2.1. If $P$ is as in Theorem 1.1 then its principal symbol $P_{m}$ can be reduced, after a multiplication by an elliptic symbol and a symplectic change of coordinates, to

$$
p(y, \eta)=\eta_{1}+i\left[y_{1}^{k_{2}-1} \eta_{2}+p_{k-1}\left(y_{1}, y_{2}\right) \eta_{n}+R\left(y, \eta^{\prime}\right)\right],
$$


near $\gamma=\left(y_{0}, \eta_{0}\right), y_{0}=0 \in \mathbb{R}^{n}$ and $\eta_{0}=(0, \ldots, 1) \in \mathbb{R}^{n}$, where $p_{k-1} \neq$ 0 is a real homogeneous polynomial of weight $k-1$ for the weights $K=$ $\left(k_{1}, k_{2}, k / 2, \ldots, k / 2, k\right)$ and the remainder $R=O_{K}\left(-\frac{1}{2}\right)$.

We recall that a function $f(y, \eta)$ is homogeneous of weight $w$ for the weights $K$ if, for $t>0$ and $(y, \eta) \in \mathbb{R}^{2 n}$, it is

$$
f\left(t^{k_{1}} y_{1}, t^{k_{2}} y_{2}, t^{k / 2} y_{3}, \ldots, t^{k} y_{n} ; t^{-k_{1}} \eta_{1}, \ldots, t^{-k} \eta_{n}\right)=t^{w} f(y, \eta) \text {. }
$$

If $h$ is a holomorphic function defined near $\gamma$, then we write $h=O_{K}(w)$ if every term in the Taylor expansion of $h$ is of weight greater than or equal to $w$ for the weights $K$.

Next, for any $\delta \in(0,1)$ we consider the following change of coordinates:

$$
\begin{aligned}
& y_{1}=\delta \tilde{y}_{1}, \\
& y_{2}=\delta^{k_{2}} \tilde{y}_{2}, \\
& y_{j}=\delta^{k / 2} \tilde{y}_{j}, \quad 3 \leq j \leq n-1, \\
& y_{n}=\delta^{k} \tilde{y}_{n} .
\end{aligned}
$$

Then the symbol $p(y, \eta)$ in $(2.2)$ is transformed to

$$
p(\tilde{y}, \tilde{\eta})=\frac{1}{\delta}\left[\tilde{\eta}_{1}+i\left[\tilde{y}_{1}^{k_{2}-1} \tilde{\eta}_{2}+p_{k-1}\left(\tilde{y}_{1}, \tilde{y}_{2}\right) \eta_{n}+\delta^{1 / k} R_{\delta}\left(\tilde{y}, \tilde{\eta}^{\prime}\right)\right]\right],
$$

where $R_{\delta}$ consists of all terms of $R$ each multiplied by a positive power of $\delta$. If we multiply $p$ by $\delta$, replace $\delta^{1 / k}$ by a new $\delta$, and drop the tildes then we obtain the following final normal form for the principal symbol of our operator $P$ :

$$
p(y, \eta)=\eta_{1}+i\left[y_{1}^{k_{2}-1} \eta_{2}+p_{k-1}\left(y_{1}, y_{2}\right) \eta_{n}+\delta R(y, \eta)\right] .
$$

Construction of phase function. Let $L$ be the vector field with symbol

$$
L(y, \eta)=\eta_{1}+i\left[y_{1}^{k_{2}-1} \eta_{2}+p_{k-1}\left(y_{1}, y_{2}\right) \eta_{n}\right] .
$$

The function

$$
Z=y_{2}+i \frac{1}{k_{2}} y_{1}^{k_{2}}
$$

satisfies the equation $L Z=0$. Also we can find real homogeneous polynomials $A\left(y_{1}, y_{2}\right)$ and $B\left(y_{1}, y_{2}\right)$ of weight $k$ for the weights $\left(k_{1}, k_{2}\right)$ such that $L W=$ 0 with

$$
W=y_{n}+B\left(y_{1}, y_{2}\right)+i A\left(y_{1}, y_{2}\right),
$$

where $A \neq 0$ and $A$ is of the form

$$
A\left(y_{1}, y_{2}\right)=c_{0} y_{1}^{k}+c_{1} y_{1}^{k-k_{2}} y_{2}+\cdots+c_{\lambda} y_{1}^{k-\lambda k_{2}} y_{2}^{\lambda}, \quad k-\lambda k_{2}>0 \text {. }
$$


We let

$$
\begin{aligned}
\varphi_{0}(x, y)= & i C\left(x_{1}-y_{1}\right)^{2}+\frac{i}{2}\left(x_{2}-Z\right)^{2} \\
& +\frac{i}{2} \sum_{j=3}^{n-1}\left(x_{j}-y_{j}\right)^{2}+\frac{i}{2}\left(x_{n}-W-a x_{2}^{k / k_{2}}\right)^{2},
\end{aligned}
$$

where $C$ is a large constant to be chosen, $a=0$ if $k / k_{2}$ is not an integer, and $a \in \mathbb{C}$ will be chosen later if $k / k_{2}$ is an integer.

The function $\varphi_{0}$ satisfies the equation

$$
\frac{\partial \varphi_{0}}{\partial x_{1}}+L \varphi_{0}=0
$$

Let $\left(x_{0}, y_{0}\right) \in \mathbb{C}^{2 n}, x_{0}=y_{0}-i \eta_{0}$, and $\left(y_{0}, \eta_{0}\right)=\gamma$ be as in (2.1). By the Cauchy-Kowalevski theorem, there is a holomorphic function $\varphi(x, y)$ defined near $\left(x_{0}, y_{0}\right)$ and such that

$$
\begin{gathered}
\frac{\partial \varphi}{\partial x_{1}}=p\left(y_{1},-\frac{\partial \varphi}{\partial y}\right)=-L \varphi+i \delta R\left(y_{1},-\frac{\partial \varphi}{\partial y^{\prime}}\right), \\
\varphi\left(0, x^{\prime}, y\right)=\varphi_{0}\left(0, x^{\prime}, y\right),
\end{gathered}
$$

where $p$ is given by (2.3). By (2.5), (2.6), and (2.7) we have

$$
\varphi(x, y)=\varphi_{0}(x, y)+O(\delta) \text {. }
$$

Let

$$
\varphi_{1}(x, y)=-\Im \varphi(x, y), \quad y \in \mathbb{R}^{n}, x \in \mathbb{C}^{n} .
$$

Then by (2.5) and (2.8) we have

$$
\begin{aligned}
\varphi_{1}(x, y)= & -C\left(y_{1}-\Re x_{1}\right)^{2}+C\left[\Im x_{1}\right]^{2} \\
& -\frac{1}{2}\left(\Re x_{2}-y_{2}\right)^{2}+\frac{1}{2}\left(\Im x_{2}-\frac{1}{k_{2}} y_{1}^{k_{2}}\right)^{2} \\
& -\frac{1}{2} \sum_{j=3}^{n-1}\left(\Re x_{j}-y_{j}\right)^{2}+\frac{1}{2} \sum_{j=3}^{n-1}\left[\Im x_{j}\right]^{2} \\
& -\frac{1}{2}\left(\Re x_{n}-y_{n}-B\left(y_{1}, y_{2}\right)-\Re\left[a x_{2}^{k / k_{2}}\right]\right)^{2} \\
& +\frac{1}{2}\left(\Im x_{n}-A\left(y_{1}, y_{2}\right)-\Im\left[a x_{2}^{k / k_{2}}\right]\right)^{2}+O(\delta) .
\end{aligned}
$$

The critical points of $\varphi_{1}(x, y)$ as a function of $y \in \mathbb{R}^{n}$ are of the form

$$
\begin{aligned}
& y_{1}=\Re x_{1}+O\left(\frac{1}{C}\right), \\
& y_{2}=\Re x_{2}-\Im x_{n} \frac{\partial A}{\partial y_{2}}\left(\Re x_{1}, \Re x_{2}\right)+\text { higher order }+O(\delta)+O\left(\frac{1}{C}\right), \\
& y_{j}=\Re x_{j}+O(\delta), \quad 3 \leq j \leq n-1, \\
& y_{n}=\Re x_{n}+B\left(\Re x_{1}, \Re x_{2}\right)+\text { higher order }+O(\delta)+O\left(\frac{1}{C}\right) .
\end{aligned}
$$


Let $\boldsymbol{\Phi}(x)$ be the critical values of $\varphi_{1}(x, y), y \in \mathbb{R}^{n}$. By (2.9) and (2.10) for $\Im x_{1}=0, x_{j}=0, j=3, \ldots, n-1$, and $x_{n}=-i$ we have

$$
\begin{aligned}
\boldsymbol{\Phi}\left(\Re x_{1}, x_{2}, 0, \ldots,-i\right)= & \frac{1}{2}\left(\Im x_{2}-\frac{1}{k_{2}}\left[\Re x_{1}\right]^{k_{2}}\right)^{2} \\
& +\frac{1}{2}+A\left(\Re x_{1}, \Re x_{2}\right)+\Im\left[a x_{2}^{k / k_{2}}\right] \\
& + \text { higher order terms }+O(\delta)+O\left(\frac{1}{C}\right) .
\end{aligned}
$$

We let

$$
\Re x_{1}=\left(k_{2} \Im x_{2}\right)^{1 / k_{2}} .
$$

By (2.11) and (2.12) we have

$$
\begin{aligned}
& \boldsymbol{\Phi}\left(\left[k_{2} \Im x_{2}\right]^{1 / k_{2}}, x_{2}, 0, \ldots,-i\right) \\
& \quad=\boldsymbol{\Phi}\left(x_{0}\right)-Q\left(x_{2}, \bar{x}_{2}\right)+\text { higher order terms }+O(\delta)+O\left(\frac{1}{C}\right),
\end{aligned}
$$

where

$$
Q\left(x_{2}, \bar{x}_{2}\right)=-A\left(\left[k_{2} \Im x_{2}\right]^{1 / k_{2}}, \Re x_{2}\right)+\Im\left[a x_{2}^{k / k_{2}}\right] .
$$

Then by (2.4) we have

$$
\begin{aligned}
Q\left(x_{2}, \bar{x}_{2}\right)= & {\left[\Im x_{2}\right]^{k / k_{2}-\lambda}\left(d_{0}\left[\Im x_{2}\right]^{\lambda}+d_{1}\left[\Im x_{2}\right]^{\lambda-1} \Re x_{2}+\cdots+d_{\lambda}\left[\Re x_{2}\right]^{\lambda}\right) } \\
& +\Im\left[a x_{2}^{k / k_{2}}\right] .
\end{aligned}
$$

We have the following

Lemma 2.2. Let $Q\left(x_{2}, \bar{x}_{2}\right)$ be as in (2.14). Then there exists an open sector $S$ in $\mathbb{C}$ with

$$
\text { angle of } S \geq \frac{\pi}{k / k_{2}} \text { and } Q\left(x_{2}, \bar{x}_{2}\right)>0 \text { on } S
$$

except possibly on a ray inside $S$, where $Q\left(x_{2}, \bar{x}_{2}\right)=0$.

Proof. Assume first that $k / k_{2}$ is an integer. Then we choose $a \in \mathbb{C}$ such that the polynomial $Q\left(x_{2}, \bar{x}_{2}\right)$ does not have harmonic terms. Thus

$$
Q\left(x_{2}, \bar{x}_{2}\right)=\left|x_{2}\right|^{2} q\left(x_{2}, \bar{x}_{2}\right), \quad \operatorname{deg} q=k / k_{2}-2 .
$$

The polynomial $Q\left(x_{2}, \bar{x}_{2}\right)$ vanishes on at most $k / k_{2}-2$ lines passing through the origin. Therefore there are at most $2\left(k / k_{2}-2\right)$ open sectors on each of which $Q$ has the same sign. Since $k$ and $k_{2}$ are odd integers, there exists an open sector $S$ such that

$$
\text { angle of } S \geq \frac{2 \pi}{2\left(k / k_{2}-2\right)}>\frac{\pi}{k / k_{2}} \text { and } Q>0 \text { in } S .
$$

Next we assume that $k / k_{2}$ is not an integer. Then let $a=0$ to get

$$
Q\left(x_{2}, \bar{x}_{2}\right)=\left(\Im x_{2}\right)^{k / k_{2}-\lambda} q_{\lambda}\left(x_{2}, \bar{x}_{2}\right),
$$


where $q_{\lambda}$ is a polynomial of degree $\lambda$. In this case we have that $q_{\lambda}$ vanishes on at most $\lambda$ lines passing through the origin. These lines form at most $2 \lambda$ open sectors on each of which $q_{\lambda}$ has the same sign. Taking into account the form of $Q$ and the fact that $k_{2}, k$ are odd numbers we conclude that there exists an open sector $S$, which might contain a ray on the line $\Im x_{2}=0$, such that angle of $S \geq 2 \pi / 2 \lambda>\pi /\left(k / k_{2}\right)$ and $Q>0$ on $S$ except possibly on $\Im x_{2}=0$, where $Q=0$. This completes the proof of Lemma 2.2.

The following lemma describes a condition on $Q$ which, as we will show later, is a sufficient condition for the operator $P$ to be analytic hypoelliptic at $\gamma$. It is an extension of Lemma III.1 in [3]. The proof given here was suggested by J. E. Fornaess.

Lemma 2.3. Let $Q\left(x_{2}, \bar{x}_{2}\right)$ be as in (2.14). Then there exist a domain $D \subset \mathbb{C}$, $0 \in D$, and a holomophic function $f\left(x_{2}\right)$ on $D$ and continuous on $\bar{D}$ with

$$
\left[Q\left(x_{2}, \bar{x}_{2}\right)+\Re f\left(x_{2}\right)\right]_{\partial D}>0, \quad f(0)=0 .
$$

Proof. Let $x_{2}=z=x+i y$. If $S$ is as in Lemma 2.2, then we can choose $\mu$ such that angle of $S>\pi / \mu>k / k_{2}$. Then after a rotation we can assume that

$$
\begin{aligned}
& Q(z, \bar{z})>0 \quad \text { if }|\arg z| \leq \frac{\pi}{2 \mu}, \quad z \neq 0, \\
& Q(z, \bar{z}) \geq 0 \quad \text { if } \arg z=\phi \text { for some } \phi,|\phi|<\frac{\pi}{2 \mu} .
\end{aligned}
$$

Let

$$
G=\{z \in \mathbb{C}:|\arg z| \leq \pi / 2 \mu,|z| \leq 1\} \cup\{0\} .
$$

For $\varepsilon>0$ we let

$$
G_{\varepsilon}=\{z-\varepsilon: z \in G\} .
$$

If $\varepsilon$ is small enough then by $(2.15)$

$$
Q(z, \bar{z}) \geq 0 \text { on }\left\{z \in G_{\varepsilon}:|z+\varepsilon|=1\right\} .
$$

To make things clear we draw a picture (Figure 1).

The region $G$ is enclosed by the segments $O_{1} A_{1}, O_{1} A_{1}^{\prime}$ and the arc $A_{1} A_{1}^{\prime}$. $G_{\varepsilon}$ is the region enclosed by the segments $O_{2} A_{2}, O_{2} A_{2}^{\prime}$ and the arc $A_{2} A_{2}^{\prime}$. The polynomial $Q(z, \bar{z})$ is nonnegative in the region $G^{\prime}$ enclosed by the segments $O_{1} A_{3}, O_{1} A_{3}^{\prime}$ and the arc $A_{3} A_{3}^{\prime}$.

Let $h$ be the harmonic extension of $-Q$ from the boundary of $G_{\varepsilon}$ to its interior, i.e., $h$ is the solution to the following Dirichlet problem:

$$
\Delta h=0, \quad h=-Q \text { on } \partial G_{\varepsilon} .
$$

We shall show that if $\varepsilon$ is small enough then

$$
h(0)<0 .
$$

If (2.17) holds then we let $f=u+i v$, where $u=h-h(0)$ and $v$ is the conjugate harmonic function of $u$ with $v(0)=0$. The function $f$ satisfies 


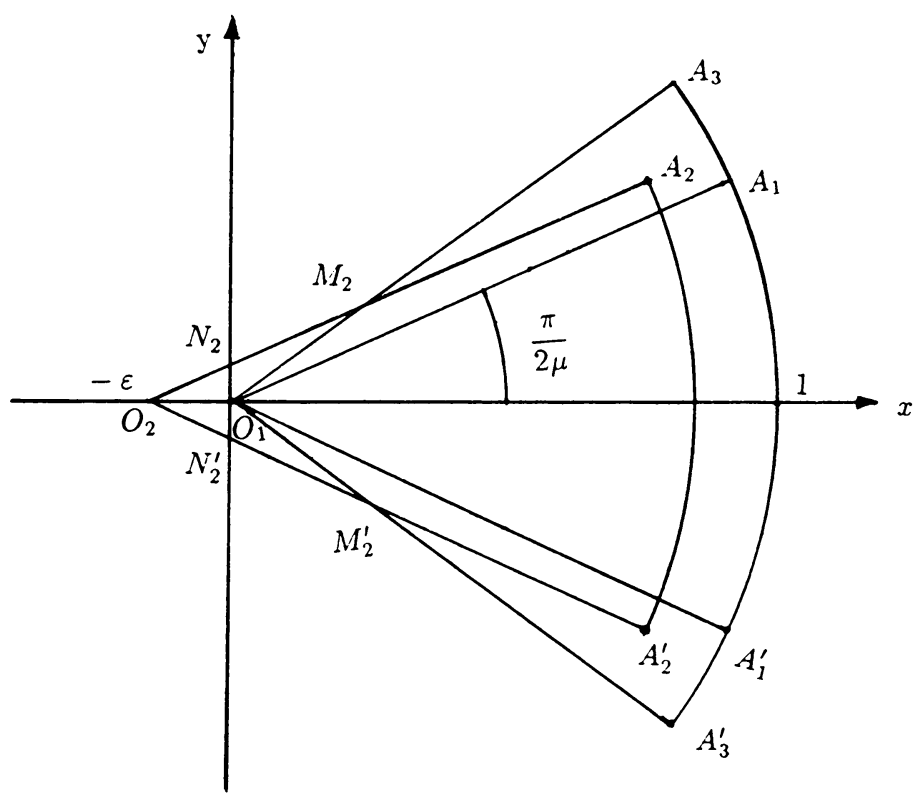

FIGURE 1

the required condition on the domain $G_{\varepsilon}$ which we choose to be the needed domain $D$.

We shall divide the boundary of $G_{\varepsilon}$ into three parts. Let $\mathbf{b}_{1}$ be the part of $\partial G_{\varepsilon}$ with $\Re z \leq 0$, i.e., the two segments $O_{2} N_{2}$ and $O_{2} N_{2}^{\prime}, \mathbf{b}_{2}$ be the part of $\partial G_{\varepsilon}$ that consists of the two segments $N_{2} A_{2}$ and $N_{2}^{\prime} A_{2}^{\prime}$, and $\mathbf{b}_{3}$ be the part of the boundary that consists of the $\operatorname{arc} A_{2} A_{2}^{\prime}$. By the homogeneity of $Q$ we have that

$$
|Q(z, \bar{z})| \leq c \varepsilon^{k / k_{2}} \quad \text { on } \mathbf{b}_{1} .
$$

Let $N_{2} M_{2}$ and $N_{2}^{\prime} M_{2}^{\prime}$ be the part of $\mathbf{b}_{2}$ that is outside $G^{\prime}$. If $z$ is on these two segments then $|z| \leq c \varepsilon$ and

$$
|Q(z, \bar{z})| \leq c|z|^{k / k_{2}} \leq c \varepsilon|z|^{k / k_{2}-1} .
$$

Since $Q$ is nonnegative on $M_{2} A_{2}$ and $M_{2}^{\prime} A_{2}^{\prime}$ it suffices to show the estimate (2.17) when

$$
|Q(z, \bar{z})| \leq c \varepsilon|z|^{k / k_{2}-1} \text { and } \quad Q<0 \text { on } \mathbf{b}_{2} .
$$

(Note. The constant $c$ is different in these inequalities and it is independent from $\varepsilon$.) Since by (2.16) $Q$ is positive on the arc $A_{2} A_{2}^{\prime}$, except possibly at one point where $Q=0$, we have that there exist a subarc $\Gamma$ of the arc $A_{2} A_{2}^{\prime}$ with

$$
0<c \leq Q(z, \bar{z}) \text { on } \Gamma \text {. }
$$




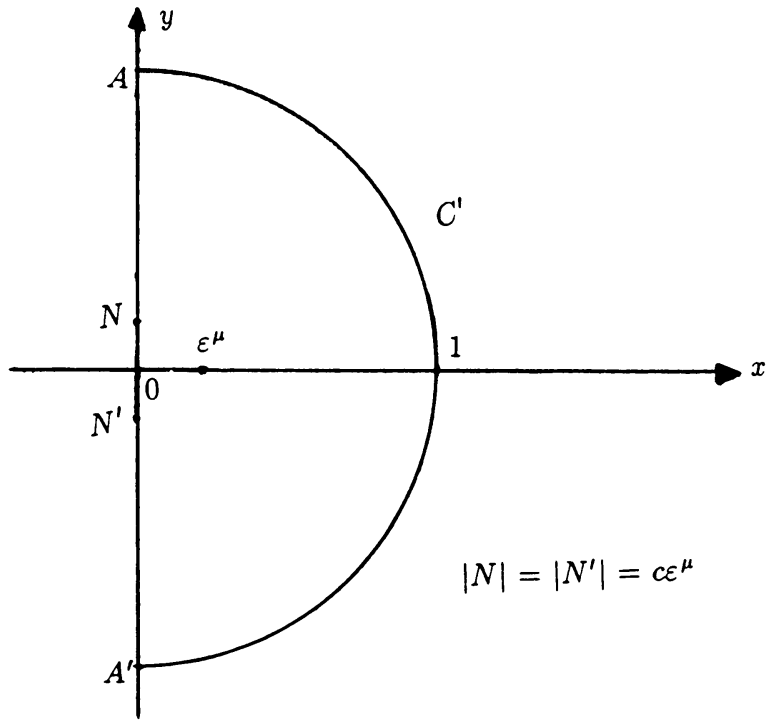

FIGURE 2

Next we map the region $G_{\varepsilon}$ onto the half unit disc by the map $z \mapsto z+\varepsilon$ followed by the map $z \mapsto z^{\mu}$. This is shown in Figure 2.

The origin is mapped to the point $\varepsilon^{\mu}, \mathbf{b}_{1}$ is mapped onto the segment $N N^{\prime}$ with $|N|=\left|N^{\prime}\right|=c \varepsilon^{\mu}, \mathbf{b}_{2}$ is mapped onto the two segments $N A$ and $N A^{\prime}$, and $\mathbf{b}_{3}$ is mapped onto the half unit circle $C^{\prime}$.

If we denote the transformed $Q$ again by $Q$ then by $(2.18)$ we have

$$
|Q(z, \bar{z})| \leq c \varepsilon^{k / k_{2}} \text { on } N N^{\prime} .
$$

By (2.19) we have

$$
|Q(z, \bar{z})| \leq c \varepsilon y^{\left(k / k_{2}-1\right) / \mu} \text { and } Q<0 \text { on } N A \text { and } N^{\prime} A^{\prime} .
$$

By (2.20) there exists an arc $\Gamma^{\prime}$ of the half circle $C^{\prime}$ such that

$$
0<c \leq Q(z, \bar{z}) \text { on } \Gamma^{\prime} \text { and } Q \geq 0 \text { on } C^{\prime} \text {. }
$$

We shall again denote by $h$ the harmonic extention of $-Q$ from the boundary of the half unit disc to the half unit disc. To show (2.17) it suffices to show that

$$
h\left(\varepsilon^{\mu}\right)<0 .
$$

To prove (2.24) we map the half unit disc conformally to the unit disc and then we use the Poisson integral

$$
h(z)=\frac{1}{2 \pi} \int_{0}^{2 \pi} \frac{1-|z|^{2}}{|\zeta-z|^{2}} u(\zeta) d \theta, \quad \zeta=e^{i \theta},
$$

for the unit disc to estimate $h\left(\varepsilon^{\mu}\right)$. 
By (2.23) the contribution $I_{3}$ to $h\left(\varepsilon^{\mu}\right)$ due to the values of $Q$ on the half circle $C^{\prime}$ is

$$
I_{3}=-c \varepsilon^{\mu}, \quad c>0 .
$$

By (2.21) the contribution to $h\left(\varepsilon^{\mu}\right)$ due to the values of $Q$ on the segment $N N^{\prime}$ is $I_{1}$ with

$$
\left|I_{1}\right| \leq c \varepsilon^{k / k_{2}}
$$

Finally by (2.22) the contribution $I_{2}$ to $h\left(\varepsilon^{\mu}\right)$ due to the values of $Q$ on the segments $N A$ and $N A^{\prime}$ is

$$
\left|I_{2}\right| \leq c \int_{\varepsilon^{\mu}}^{1} \frac{\varepsilon^{\mu}}{y^{2}+\varepsilon^{2 \mu}} \varepsilon y^{\left(k / k_{2}-1\right) / \mu} d y \leq c \varepsilon^{1+\mu} \int_{\varepsilon^{\mu}}^{1} y^{\left(k / k_{2}-1\right) / \mu-2} d y .
$$

Since $\mu$ can be chosen such that $k / k_{2}=\mu+\alpha$, with $0<\alpha<1$, the last integral is equal to

$$
\int_{\varepsilon^{\mu}}^{1} \frac{1}{y^{1+(1-\alpha) / \mu}} d y=-\frac{\mu}{1-\alpha}\left[1-\frac{1}{\varepsilon^{1-\alpha}}\right] .
$$

Therefore,

$$
\left|I_{2}\right| \leq c_{1} \varepsilon^{1+\mu}+c_{2} \varepsilon^{\mu+\alpha}, \quad 0<\alpha<1 .
$$

By (2.25), (2.26), and (2.27) we see that $I_{3}$ is the dominant term for $\varepsilon>0$ and small enough. Therefore inequality (2.24) holds and this completes the proof of Lemma 2.3.

Lemma 2.3 provides a sufficient condition for the p.d.o. $P$ to be analytic hypoelliptic at $\gamma$ (see $[3,6]$ ). In the following we give an outline of the proof of this fact.

Let $\left(x_{0}, y_{0}\right) \in \mathbb{C}^{2 n}$ with

$$
x_{0}=y_{0}-i \eta_{0}, \quad\left(y_{0}, \eta_{0}\right)=\gamma \text { is given by }(2.1) .
$$

We can find an elliptic classical analytic symbol $a$ near $\left(x_{0}, y_{0}\right)$ (see [9]) such that the Fourier-Bros-Iagolnitzer transformation defined by

$$
(T u)(x, \lambda)=\int_{\mathbb{R}^{n}} e^{i \lambda \varphi(x, y)} a(x, y, \lambda) \chi(y) u(y) d y, \quad \lambda>0,
$$

with $\chi \in C_{0}^{\infty}\left(\mathbb{R}^{n}\right), \chi=1$ near $y_{0}$, and $\varphi$ given by (2.7) reduces the operator $P$, with principal symbol given by $(2.3)$, to complex $D_{x_{1}}$; i.e., there exists an $\varepsilon_{1}>0$ such that

$$
\left|\left(\frac{1}{\lambda} D_{x_{1}} T-T P\right) u(x, \lambda)\right| \lesssim e^{\lambda\left[\Phi(x)-\varepsilon_{1}\right]} \text { near } x_{0}
$$


Now let $u$ be a distribution defined near $y_{0}$ and such that $P u$ is analytic at $\gamma$. By (2.29) it follows that there exists $\varepsilon_{2}>0$ such that

$$
\left|D_{x_{1}} T u(x, \lambda)\right| \lesssim e^{\lambda\left[\Phi(x)-\varepsilon_{2}\right]} \text { near } x_{0} .
$$

To prove Theorem 1.1 it suffices to show that there exists $\varepsilon_{3}>0$ such that

$$
|T u(x, \lambda)| \lesssim e^{\lambda\left[\Phi(x)-\varepsilon_{3}\right]} \text { near } x_{0} .
$$

Relation (2.31) follows from (2.30) and Lemma 2.3. In fact, by (2.13) and Lemma (2.3) we have that there exists $\varepsilon_{4}>0$ such that

$$
\left[\Phi\left(\left[k_{2} \Im x_{2}\right]^{1 / k_{2}}, x_{2}, 0, \ldots,-i\right)-\Re f\left(x_{2}\right)\right]_{\partial D}<\Phi\left(x_{0}\right)-\varepsilon_{4},
$$

for $C$ large enough and $\delta$ small enough. Notice also that for (2.32) to hold we may need to replace $D$ by a dilation of $D$.

By (2.32) we have

$$
\Phi\left(\left[k_{2} \Im x_{2}\right]^{1 / k_{2}}, x^{\prime}\right)-\Re f\left(x_{2}\right)<\Phi\left(x_{0}\right)-\varepsilon_{4} / 2
$$

if $x_{2} \in \partial D$ and $\left|x^{\prime \prime}-x_{0}^{\prime \prime}\right|<r$ for some $r>0$, where $x^{\prime \prime}=\left(x_{3}, \ldots, x_{n}\right)$.

By (2.30) and the fact that $f(0)=0$ we have

$$
\left|D_{x_{1}}\left[e^{-\lambda f\left(x_{2}\right)} T u(x, \lambda)\right]\right| \lesssim e^{\lambda\left[\Phi(x)-\varepsilon_{2} / 2\right]} \text { near } x_{0} .
$$

By (2.33) we have

$$
\left|e^{-\lambda f\left(x_{2}\right)} T\left(\left[k_{2} \Im x_{2}\right]^{1 / k_{2}}, x^{\prime}, \lambda\right)\right| \lesssim e^{\lambda\left[\Phi\left(x_{0}\right)-\varepsilon_{4} / 2\right]}
$$

if $x_{2} \in \partial D$ and $\left|x^{\prime \prime}-x_{0}^{\prime \prime}\right|<r$.

By (2.34), (2.35), and an integration along $x_{1}$ we have that there exists $\varepsilon_{5}$ such that

$$
\left|e^{-\lambda f\left(x_{2}\right)} T u\left(0, x^{\prime}, \lambda\right)\right| \lesssim e^{\lambda\left[\Phi\left(x_{0}\right)-\varepsilon_{5}\right]},
$$

where $x_{2} \in \partial D$ and $\left|x^{\prime \prime}-x_{0}^{\prime \prime}\right|<r_{1}$, for some $r_{1}>0$.

Now we apply the maximum principle in $x_{2}$ in $D$ and we use (2.36) to obtain

$$
\left|e^{-\lambda f\left(x_{2}\right)} T u\left(0, x^{\prime}, \lambda\right)\right| \lesssim e^{\lambda\left[\Phi\left(x_{0}\right)-\varepsilon_{5}\right]}, \quad x_{2} \in D,\left|x^{\prime \prime}-x_{0}^{\prime \prime}\right|<r_{1} .
$$

By (2.34), (2.37), and an integration along $x_{1}$ we get

$$
\left|e^{-\lambda f\left(x_{2}\right)} T u(x, \lambda)\right| \lesssim e^{\lambda\left[\Phi\left(x_{0}\right)-\varepsilon_{6}\right]} \text { near } x_{0} \text { for some } \varepsilon_{6}>0 .
$$

Since $f(0)=0$, relation $(2.38)$ implies that

$$
|T u(x, \lambda)| \lesssim e^{\lambda\left[\Phi\left(x_{0}\right)-\varepsilon_{6} / 2\right]} \text { near } x_{0} .
$$

By (2.39) we obtain (2.31) which completes the proof of Theorem 1.1. 


\section{REFERENCES}

1. M. S. Baouendi and L. P. Rothschild, Cauchy-Riemann functions on manifolds of higher codimension in complex space, preprint, 1989.

2. __ Normal forms for generic manifolds and holomorphic extension of $C R$ functions, $\mathbf{J}$. Differential Geom. 25 (1987), 431-467.

3. M. S. Baouendi, L. P. Rothschild, and F. Treves, $C R$ structures with group action and extendability of $C R$ functions, Invent. Math. 82 (1985), 359-467.

4. Y. V. Egorov, Subelliptic pseudo-differential operators, Dokl. Akad. Nauk SSSR 188 (1969), 20-22.

5. A. A. Himonas, On analytic microlocal hypoellipticity of linear partial differential operators of principal type, Comm. Partial Differential Equations 11 (1986), 1539-1574.

6. $\ldots$ Semirigid partial differential operators and microlocal analytic hypoellipticity, Duke Math. J. 59 (1989), 265-287.

7. L. Hörmander, Subelliptic operators, Ann. of Math. Studies, no. 91, Princeton Univ. Press, Princeton, N.J., 1979, pp. 127-208.

8. J. J. Kohn, Boundary behavior of $\bar{\partial}$ on weakly pseudoconvex manifolds of dimension two, J. Differential Geom. 6 (1972), 523-542.

9. J. Sjöstrand, Singularités analytiques microlocales, Astérisque 95, Soc. Math. France, 1982.

10. A. E. Tumanov, Extending $C R$ functions on manifolds of finite type to a wedge, Mat. Sb. 136 (1988), 128-139. (Russian)

Department of Mathematics, University of Notre Dame, Notre Dame, Indiana 46556 